\title{
"Peer Support" intervention to improve glycemic control in type 2 diabetes mellitus: A literature review
}

\author{
Hasrima $^{1,2, *}$, Elly Lilianty Sjattar², Andi Masyitha Irwan² \\ ${ }^{1}$ Karya Kesehatan Institute of Health Science, Kendari, South-East Sulawesi, Indonesia \\ ${ }^{2}$ Medical Surgical Nursing Departments, Nursing Faculty, Hasanuddin University, South Sulawesi, Indonesia
}

\begin{abstract}
SUBMITTED: 15 December 2020 REVISED: 15 January 2021 ACCEPTED: 22 February 2021
\end{abstract}
KEYWORDS

Diabetes mellitus type 2

Glycemic control

Peer support

\begin{abstract}
Hyperglycemia is one of the complications of Type 2 Diabetes Mellitus (T2DM). Continuation of this situation in T2DM will lead to more serious complications such as morbidity and death. There are improvements and advances in the management of T2DM at present, but they are accompanied by an increase in medical costs. To solve these problems, an organized approach is needed to control hyperglycemia through peer support interventions which are considered to be able to reduce treatment costs. Peer support may take advantage of the ability of diabetes patients to support each other in managing their daily lives, which is very useful as a step to promote the type of daily behavioral efforts needed to prevent disease progression. This study aimed to identify the effect of peer support intervention in improving glycemic control in patients with T2DM. This study was a literature review using seven databases including PubMed, ScienceDirect, ProQuest, Clinical Key, Cochrane, Ebsco, and DOAJ. Inclusion criteria were: articles published in the last 10 years, in full text and in English version. Five studies were reviewed that met the inclusion criteria. The results of this review showed the effectiveness of peer support in improving glycemic control in diabetes patients. Peer support significantly lowered HbA1c and also led to a significant reduction in fasting blood sugar. Peer support may reduce glycosylated blood hemoglobin levels, increase knowledge regarding insulin use and increase diabetes self-management skills, especially in patients who do not have literacy skills. In conclusion, peer support can be used as a non-pharmacological therapy to improve glycemic control in patients with T2DM.
\end{abstract}

(c) The Journal 2021. This article is distributed under a Creative Commons Attribution-ShareAlike 4.0 International license.

\section{Introduction}

Diabetes mellitus is a chronic disease with a prevalence of more than 300 million people worldwide. ${ }^{1}$ Globally, the prevalence of diabetes sufferers will increase in the years to come and Indonesia is predicted to increase by 212 million sufferers by $2045 .^{2}$ One of the most common types is type 2 diabetes mellitus (T2DM), where this type occurs due to insulin resistance resulting in an increase in blood sugar levels ${ }^{3}$, which then becomes hyperglycemic.

Hyperglycemia is a complication of T2DM which is at risk of a metabolic emergency and will result in morbidity. ${ }^{4}$ The increase in the prevalence of T2DM is in line with the increase in hyperglycemic cases which

\footnotetext{
*Correspondence: hasrima85@gmail.com

Karya Kesehatan Institute of Health Science, Jl. Jend. $A H$. Nasution No.89, Anduonohu, Kec. Kambu, Kota Kendari, Sulawesi Tenggara, Indonesia 93561
}

implies an increase in the cost of care. ${ }^{5}$ Although there has been progress in terms of diabetes management, it turns out that with the low awareness of T2DM sufferers in glycemic control and in managing their own disease, they still face serious challenges. ${ }^{6}$

Given the increasing prevalence of T2DM and the lack of diabetes educators, these concerns become the basis for integrating and using lowcost interventions in high-risk communities through the empowerment of available resources. ${ }^{7}$ In order to overcome this problem, an approach is needed to minimize complications, reduce medical costs and improve patients' quality of life. ${ }^{8}$ Therefore, hyperglycemia demands the appropriate approach and treatment plan to reduce morbidity and mortality. ${ }^{4}$

Several interventions have been developed in an effort to improve glycemic control but the results have not been optimal. Peer support can be applied 
through the use of sufferers' abilities to support each other in managing their daily life, This strategy is also useful as a step to promote types of daily behavioral efforts $^{9}$ through meetings between sufferers and exchanging information about health problems ${ }^{10}$, solving health problems and providing emotional support. ${ }^{11}$

Emotional support in the peer support program motivates patients to improve glycemic control ${ }^{12}$ and will ultimately minimize treatment costs. ${ }^{12}$ Peer support has been shown to improve glycemic control in T2DM patients. ${ }^{13-15}$ Therefore, it is necessary to conduct a review related to peer support program intervention in improving glycemic control in patients with T2DM. Thus, the aim of this review was to describe peer support interventions in improving glycemic control in T2DM patients.

\section{Method}

This literature review uses the PRISMA 2009 guidelines for a systematic review ${ }^{16}$ and available at http://prismastatement.org/PRISMAStatement/ Checklist.aspx. The literature search used the
PubMed, ScienceDirect, Proquest, Clinical key, Cochrane, Ebsco and DOAJ databases with restrictions on articles published in 2010 to 2020 . Question formulations were based on PICO (patient, intervention, comparison / control and outcome), in detail P: Patients with type 2 Diabetes mellitus, I: Peer Support, C: Standard care, usual care, and O: glycemic control.

All potentially eligible studies were considered, and full papers were reviewed to determine whether they met the selection criteria. The criteria included in this review were patients with T2DM, focused only on peer support interventions, glycemic control was primary and secondary outcomes and the 2010-2020 study.

Screening results from the seven databases used found 109 articles identified with all human subjects. We excluded 151 duplications, 1,128 irrelevant to the research question, 4 not available full texts, 5 not in English, 7 qualitative studies, 5 not a study, and 4 using additional interventions. Thus, only five intervention articles met the inclusion criteria as described in Figure 1.

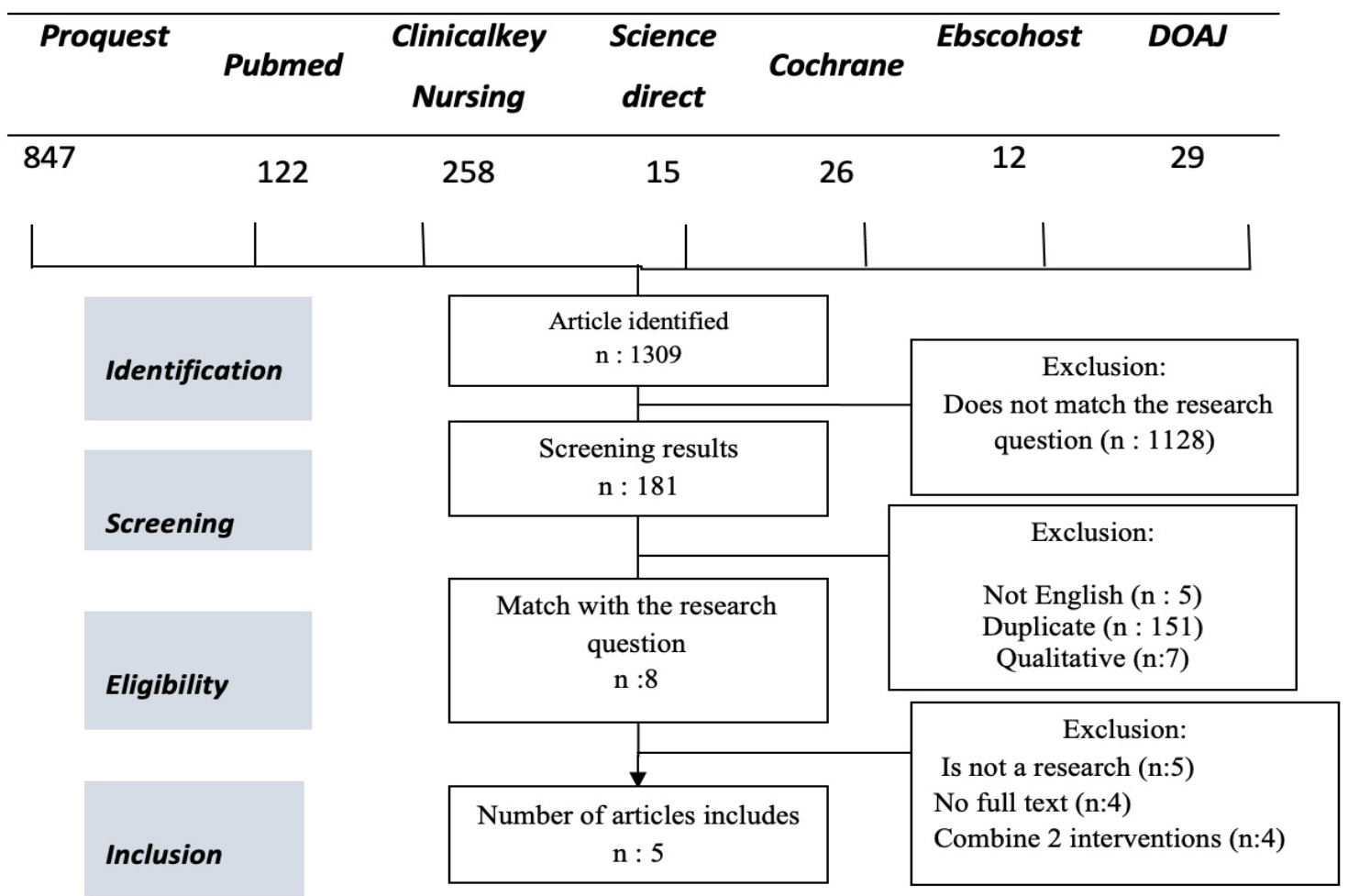

Figure 1. Flowchart 


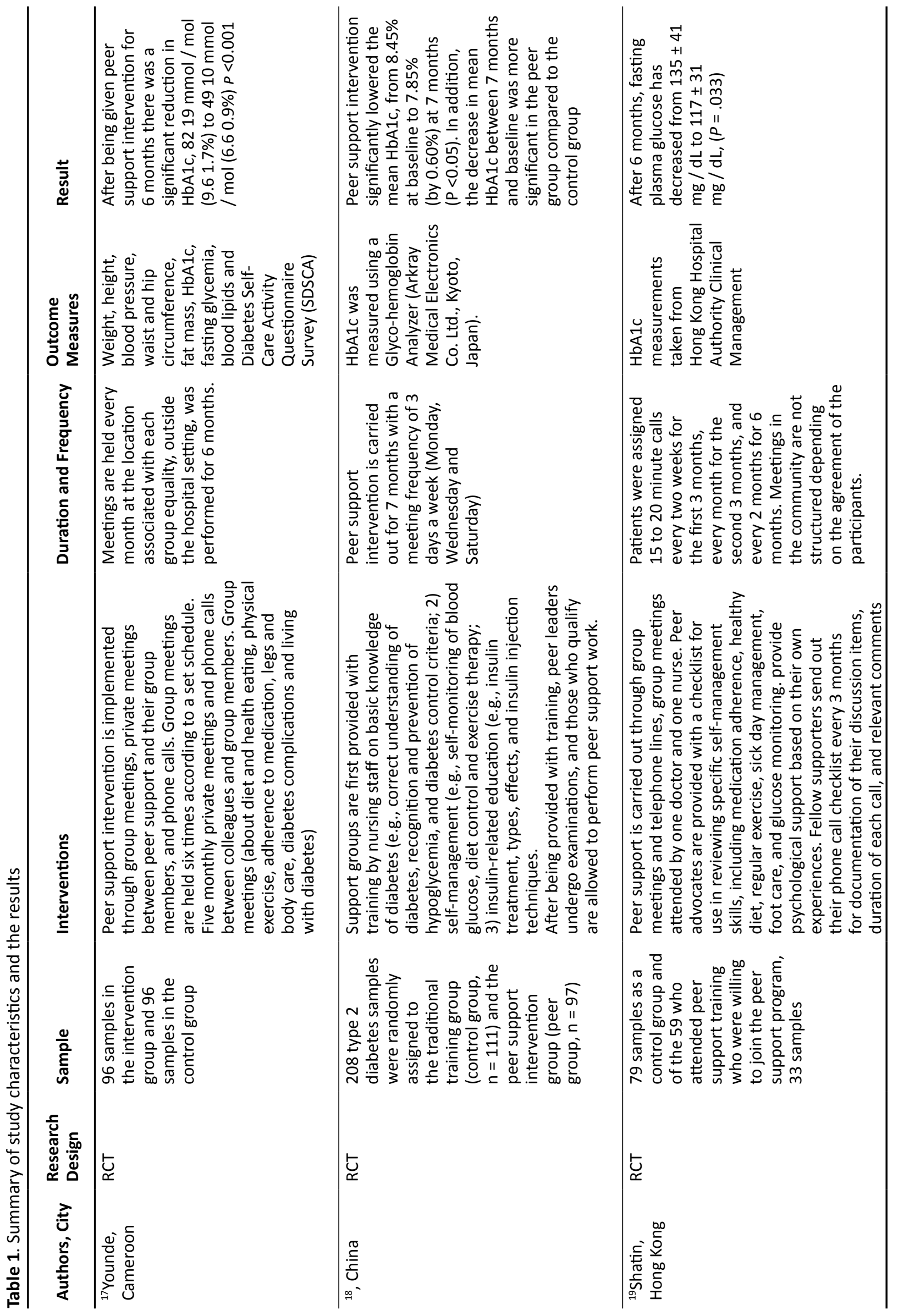




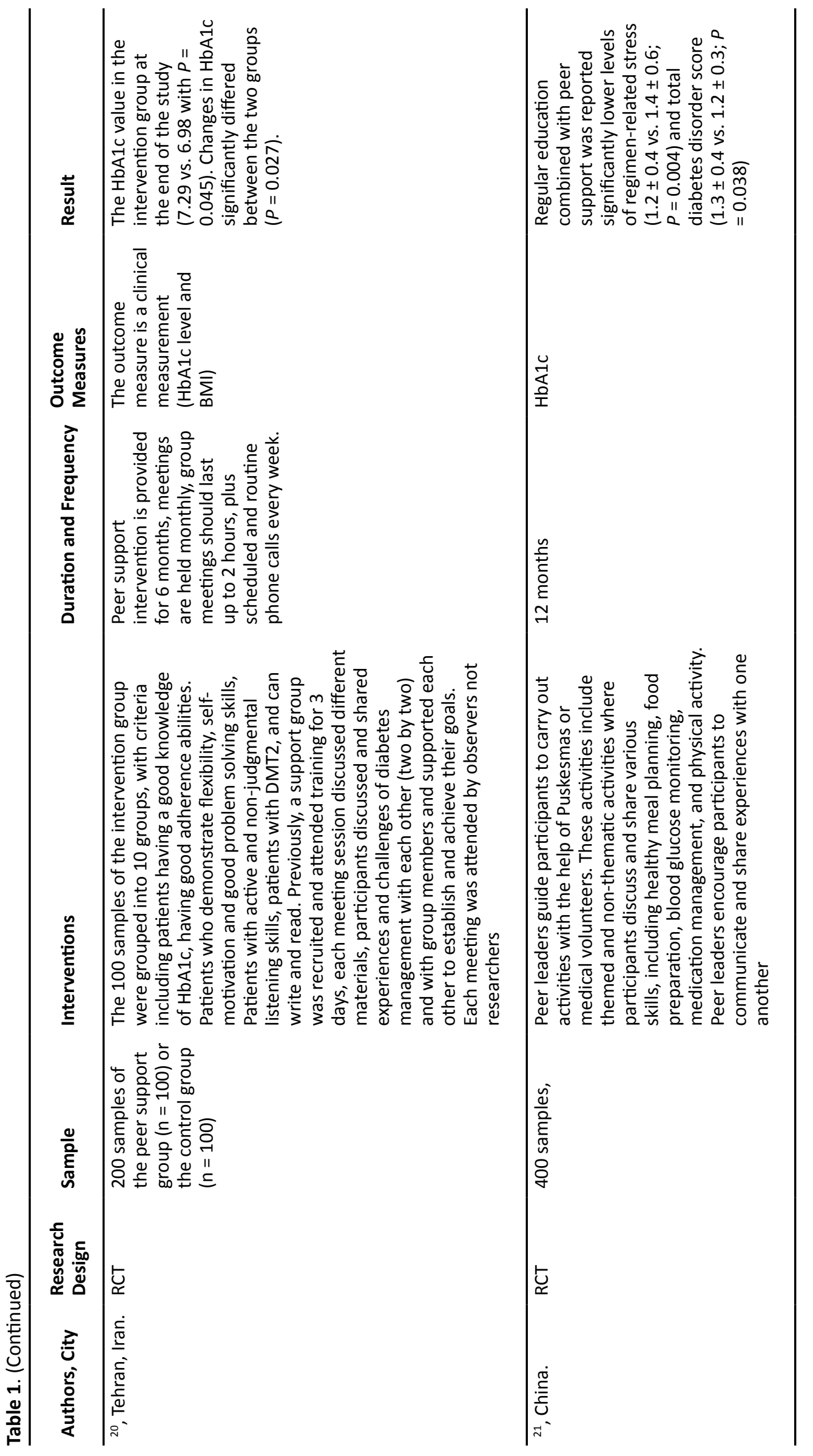




\section{Result}

Five articles that meet the criteria that have been reviewed are summarized in Table 1 . These articles were published in 2010-2020 with a randomized control trial research design.

\subsection{Characteristics of the study}

Study characteristics are presented in Table 1. All studies using the RCT methods provide peer support intervention. ${ }^{17-21}$ The peer support method is carried out through group meetings, with 2 articles combining group meetings with telephone calls. ${ }^{17,19}$ Before implementing peer support, peer leaders are encouraged to take part in training guided by health workers. ${ }^{18,21}$ Peer leaders were invited to volunteer and underwent 2 days of workshops to strengthen their knowledge and skills to support diabetics, strengthen participants' knowledge about diabetes, training on communication skills. ${ }^{17}$ In addition, supporters are recruited and participate in training for 3 days $^{20}$. Meanwhile, the peer leaders are selected based on residence, demographics, and other characteristics, including interpersonal skills assessed at interview sessions, willingness to work together as part of a team and follow study protocol. $^{19}$

The group meetings were designed to discuss healthy diet and food, physical exercise, medication adherence, body and foot care, diabetes complications and living with diabetes ${ }^{17}$ selfmanagement skills, healthy diet, regular exercise, and glucose monitoring. Participants are also encouraged to provide psychological support based on their own experiences $^{19}$ sharing diabetes knowledge (e.g., understanding diabetes, recognition and prevention of hypoglycemia), self-management (e.g., monitoring their own blood glucose, control of diet and exercise) related to insulin knowledge (e.g., insulin treatment, types, effects, and insulin injection techniques). ${ }^{18}$

In addition, participants discuss problems and concerns that affect their adherence to medication. In the second session, they discussed their views on difficulties in adhering to diet and diabetics' perceptions of obesity risk and weight control ${ }^{20}$ and motivated each other to maximize diabetes self-care management. ${ }^{21}$ Of the five articles that were included, it was shown that peer support can significantly reduce $\mathrm{HbA} 1 \mathrm{c}[-33 \mathrm{mmol} / \mathrm{mol}(-3.0 \%)]$ $\mathrm{P}<0.001^{17}$, After 6 months, patients in the peer support group experienced a significant decrease in the mean A1c value of $P=0.045^{20}$ Thus, patients with T2DM who are continuously involved in peer support programs improve their self-care and maintain glycemic control. ${ }^{19}$

\section{Discussion}

Based on the five studies we reviewed, all studies using the RCT research design showed that peer support interventions were effective in improving glycemic control in T2DM patients, complementing pharmacological treatment. Thus, these results have important implications for current public health practice. Peer support is a protection for a wide range of activities carried out. This form of activity is done continuously which includes how to solve problems. ${ }^{22} \mathrm{~A}$ study revealed a significant increase in glycemic control through the application of peer support interventions that support each other in DMT2 management behavior. ${ }^{23}$ Support through clinical tele-monitoring applications has also resulted in important improvements in glycemic control in patients with T2DM in family practice..$^{24}$

Peer support programs are an acceptable way to provide additional care to support adherence and treatment management, especially in situations where there is a shortage of health workers, ${ }^{25}$ after 12 months of peer support intervention 94\% of participants said the program has important implications for controlling their glycemic load. ${ }^{26}$ It can also result in weight loss and improve patients' quality of life. ${ }^{27}$

Peer support intervention had a greater impact on $\mathrm{A} 1 \mathrm{C}$ among patients actively involved in the peer support group ( $p<0.01)$. This was evaluated after participating in a peer support group for 12 months which was divided into two sessions including 6 months with group meetings, and 6 months continued by telephone, there was a significant decrease in $\mathrm{A} 1 \mathrm{C} .{ }^{11}$ This peer support model may have a positive impact on diabetic disorders, therefore it is necessary to modify culture responsiveness (e.g., 
intervention sites) to a pilot model to produce better health outcomes. ${ }^{28}$

\section{Conclusion}

A literature review related to peer support interventions for glycemic control reveals that in general, peer support interventions with various approaches are considered effective in improving glycemic control in T2DM patients. Accordingly, it can be used as a complement to conventional therapy. Therefore, peer support interventions are feasible to be applied in a clinical setting. To confirm these findings, further research is needed.

\section{Acknowledgement}

This study did not receive specific grand funding agencies in the public sector, commercial, or nonprofit sector.

\section{Conflict of interests}

There is no conflict of interest.

\section{References}

1. Sugiarta IGRM, Darmita IGK. Profil penderita Diabetes Mellitus Tipe-2 (DM-2) dengan komplikasi yang menjalani rawat inap di Rumah Sakit Umum Daerah (RSUD) Klungkung, Bali tahun 2018. Intisari Sains Medis [Internet]. 2020;11(1):7-12. Available from: http:// isainsmedis.id/

2. International Diabetes Federation. IDF Diabetes Atlas Ninth edition 2019 [Internet]. International Diabetes Federation. 2019. 176 p. Available from: http://www.idf.org/about-diabetes/factsfigures

3. Bhatt H, Saklani S, Upadhayay K. Anti-oxidant and anti-diabetic activities of ethanolic extract of Primula Denticulata Flowers. Indones J Pharm. 2016;27(2):74-9.

4. Van Ness-Otunnu R, Hack JB. Hyperglycemic crisis. J Emerg Med. 2013;45(5):797-805.

5. Nyenwe EA, Kitabchi AE. Evidence-based management of hyperglycemic emergencies in diabetes mellitus. Diabetes Res Clin Pract. 2011;94(3):340-51.
6. L. DV, a.a.W.a. VDH, E. V'T R, C.a. B, P.J. K, M. $R$, et al. Peer support to decrease diabetesrelated distress in patients with type 2 diabetes mellitus: design of a randomised controlled trial. BMC Endocr Disord. 2014;14:1-6.

7. Piatt GA, Rodgers EA, Xue L, Zgibor JC. Integration and utilization of peer leaders for diabetes selfmanagement support: Results from project SEED (Support, Education, and Evaluation in Diabetes). Diabetes Educ. 2018;44(4):373-82.

8. Duncan BB, Schmidt MI, Ewerton Cousin, Moradi-Lakeh M, Passos VMDA, França EB, et al. The burden of diabetes and hyperglycemia in Brazil-past and present: findings from the Global Burden of Disease Study 2015. Diabetol Metab Syndr. 2017;9(1):1-12.

9. Fisher BE, Boothroyd IR, Coufal MM, Baumann $\mathrm{CL}$, Mbanya JC, Rotheram-Borus MJ, et al. Peer support for self-management of diabetes improved outcomes in international settings. Health Aff. 2012;31(1):130-9.

10. Paul G, Keogh K, D'Eath M, Smith SM. Implementing a peer-support intervention for people with type 2 diabetes: a qualitative study. Fam Pract. 2013;30(5):593-603.

11. Piette JD, Resnicow K, Choi H, Heisler M. A diabetes peer support intervention that improved glycemic control: mediators and moderators of intervention effectiveness. Chronic IIIn. 2013;9(4):258-67.

12. Thankappan KR, Sathish T, Tapp RJ, Shaw JE, Lotfaliany $M$, Wolfe $R$, et al. A peer-support lifestyle intervention for preventing type 2 diabetes in India: A cluster-randomized controlled trial of the Kerala Diabetes Prevention Program. PLoS Med. 2018;15(6):1-23.

13. Zhang $X$, Yang $S$, Sun $K$, Fisher EB, Sun X. How to achieve better effect of peer support among adults with type 2 diabetes: a meta-analysis of randomized clinical trials. Patient Educ Couns. 2016;99(2):186-97.

14. Dale JR, Williams SM, Bowyer V. What is the effect of peer support on diabetes outcomes in adults? A systematic review. Diabet Med. 2012;29(11):1361-77.

15. Qi L, Liu Q, Qi X, Wu N, Tang W, Xiong $H$. Effectiveness of peer support for improving 
glycaemic control in patients with type 2 diabetes: a meta-analysis of randomized controlled trials. BMC Public Health. 2015;15(1).

16. Moher D, Liberati A, Tetzlaff J, Altman DG. Preferred Reporting Items for Systematic Reviews and Meta-Analyses: the PRISMA statement. Ann Intern Med. 2009;151(4):264-9.

17. Assah FK, Atanga EN, Enoru S, Sobngwi E, Mbanya JC. Community-based peer support significantly improves metabolic control in people with type 2 diabetes in Yaoundé, Cameroon. Diabet Med. 2015;32(7):886-9.

18. Deng K, Ren Y, Luo Z, Du K, Zhang X, Zhang Q. Peer support training improved the glycemic control, insulin management, and diabetic behaviors of patients with type 2 diabetes in rural communities of Central China: a randomized controlled trial. Med Sci Monit. 2016;22:26775.

19. Yin J, Wong R, Au S, Chung $H$, Lau M, Lin L, et al. Effects of providing peer support on diabetes management in people with type 2 diabetes. Ann Fam Med. 2015;13:S42-9.

20. Peimani M, Monjazebi F, Ghodssi-Ghassemabadi R, Nasli-Esfahani E. A peer support intervention in improving glycemic control in patients with type 2 diabetes. Patient Educ Couns. 2018;101(3):460-6.

21. Ju C, Shi R, Yao L, Ye X, Jia M, Han J, et al. Effect of peer support on diabetes distress: a cluster randomized controlled trial. Diabet Med. 2018;35(6):770-5.

22. Support P. Peer Support in Action: From Bystanding to Standing by. Peer Support Action From Bystanding to Standing by. 2012;

23. Sreedevi A, Gopalakrishnan UA, Karimassery Ramaiyer S, Kamalamma L. A Randomized controlled trial of the effect of yoga and peer support on glycaemic outcomes in women with type 2 diabetes mellitus: a feasibility study. BMC Complement Altern Med. 2017;17(1):1-8.

24. Wild SH, Hanley J, Lewis SC, McKnight JA, McCloughan LB, Padfield PL, et al. Supported telemonitoring and glycemic control in people with type 2 Diabetes: The telescot diabetes pragmatic multicenter randomized controlled trial. PLoS Med. 2016;13(7):1-16.
25. Akena D, Okello ES, Simoni J, Wagner G. The development and tailoring of a peer support program for patients with diabetes mellitus and depression in a primary health care setting in Central Uganda. BMC Health Serv Res. 2020;20(1):1-9.

26. Aziz Z, Riddell MA, Absetz P, Brand M, Oldenburg $B$, Dunbar JA, et al. Peer support to improve diabetes care: an implementation evaluation of the Australasian Peers for Progress Diabetes Program. BMC Public Health. 2018;18(1):1-12.

27. Khodneva Y, Safford MM, Richman J, Gamboa C, Andreae S, Cherrington A. Volunteer peer support, diabetes, and depressive symptoms: results from the ENCOURAGE trial. J Clin Transl Endocrinol. 2016;4:38-44.

28. Tang TS, Sohal PS, Garg AK. Rethinking peer support for diabetes in Vancouver's South-Asian community: a feasibility study. Diabet Med. 2015;32(8):1077-84. 